\title{
Propagation of Double Resonance and Dark Area
}

\author{
J.H. EBERLY ${ }^{a}$ AND V.V. KOZLOV ${ }^{a, b}$ \\ ${ }^{a}$ Department of Physics and Astronomy and Rochester Theory Center \\ for Optical Science and Engineering \\ University of Rochester, Rochester, New York 14627, USA \\ ${ }^{b}$ Abteilung für Quantenphysik, Universität Ulm, 89069, Germany
}

\begin{abstract}
We report new results in propagation of two coherently coupled optical pulses in an inhomogeneously broadened three-level lambda medium.

PACS numbers: $42.50 .-\mathrm{p}, 42.50 . \mathrm{Dv}, 42.50 . \mathrm{Lc}, 42.50 . \mathrm{Gy}$
\end{abstract}

A wide variety of quantum optical coherence effects, as well as experimental techniques, are based on double resonance. However, spatial propagation of two fields that are coupled in a double-resonance interaction has not previously been investigated. This may be important in light of the increasing use of double resonance in new effects such as lasing without inversion (LWI) and electromagnetically induced transparency (EIT) [1,2].

The area theorem for evolution of a coherent pulse in an inhomogeneously broadened two-level medium derived by McCall and Hahn in 1967 plays a central role in the nonlinear optical theory of resonant systems [3, 4]. It provides us with a unique intuitive guide through complex matter-field behavior, relying only on the knowledge of the initial value of the pulse's area. The McCall-Hahn area theorem stands on such solid physical ground that its predictions extend beyond the regime where its derivation has a rigorous character (see [5]).

It would be attractive to have a theorem of similar generality that can be applied for two fields interacting with two different resonant transitions. The most straightforward approach is implemented in $[6,7]$, where area theorems are derived for $V$ and $A$ systems under the restriction of initially identical pulse shapes, in which case the three-level media exhibit only familiar two-level behavior. Another, nontraditional, approach would be to step away from the two-level philosophy by focusing on those elements specific to three-level systems with no analogy in two-level physics, namely the physics of the well-known "dark state" [8]. 
The most attractive features of three-level systems occur in a $A$ configuration as shown in Fig. 1a. The long-lived stability of the two lower states gives rise to numerous effects ranging from coherent population trapping [9], to counterintuitive excitation [10], matched pulse propagation [11], adiabatons [12], and many others. The dark state of lambda systems manifests itself as a coherent superposition of the ground levels which is decoupled from the incident fields by destructive interference of the absorption paths.
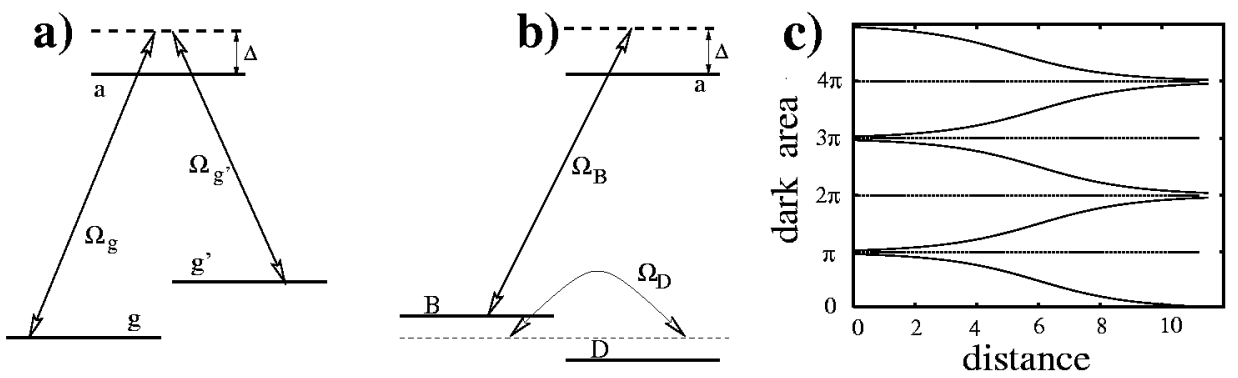

Fig. 1. (a) and (b) Sketches of the bare-state and bright/dark-state pictures of a typical atom in a $\Lambda$-type medium. (c) Dark area $\theta_{\mathrm{D}}$ versus distance for $\phi=0$, see Eq. (4). Distance is measured in Beer's lengths, $\alpha \zeta$.

Evolution equations for a generic lambda configuration can be written using the standard travelling-wave coordinates $\zeta=z$, and $\tau=t-z / c$, the local time in a reference frame moving with the velocity of light in the medium

$$
\begin{aligned}
& \frac{\partial}{\partial \zeta} \Omega_{g}=\mathrm{i} \mu\left\langle a g^{*}\right\rangle, \quad \frac{\partial}{\partial \zeta} \Omega_{g^{\prime}}=\mathrm{i} \mu^{\prime}\left\langle a g^{\prime *}\right\rangle, \\
& \mathrm{i} \frac{\partial}{\partial \tau} g=-\frac{1}{2} \Omega_{g}^{*} a, \quad \mathrm{i} \frac{\partial}{\partial \tau} g^{\prime}=-\frac{1}{2} \Omega_{g^{\prime}}^{*} a, \quad \mathrm{i} \frac{\partial}{\partial \tau} a=\Delta a-\frac{1}{2}\left(\Omega_{g^{\prime}} g^{\prime}-\Omega_{g} g\right) .
\end{aligned}
$$

The notation involving Rabi frequencies $\Omega$ and probability amplitudes $\left(g, g^{\prime}, a\right)$ is conventional (see Fig. 1a). We assume that the fields are initially unchirped and remain so. The angular brackets in (1) refer to the Doppler averages. We do not require $\mu=\mu^{\prime}$ for the two coupling constants but we treat only that case here [13].

The usual dressed basis follows from a rotation in $g-g^{\prime}$ space:

$$
\left[\begin{array}{l}
B \\
D
\end{array}\right]=\left[\begin{array}{cc}
\cos \theta / 2 & \sin \theta / 2 \\
-\sin \theta / 2 & \cos \theta / 2
\end{array}\right]\left[\begin{array}{l}
g \\
g^{\prime}
\end{array}\right],
$$

where the dressing angle $\theta$ is defined (up to $\pm n \pi$ ) by $\cos \theta / 2 \equiv \Omega_{g} / \Omega_{\mathrm{B}}$ and $\sin \theta / 2 \equiv$ $\Omega_{g^{\prime}} / \Omega_{\mathrm{B}}$, and the auxiliary "bright field" is defined as $\Omega_{\mathrm{B}} \equiv \sqrt{\Omega_{g}^{2}+\Omega_{g^{\prime}}^{2}}$, and the bright-dark coupling scheme is indicated in Fig. 1b. It is already known [14] that $\theta$ 
is important for temporal evolution: the magnitude of $\dot{\theta}$ separates non-adiabatic from adiabatic regimes.

There are unexpected advantages in treating the dressing angle $\theta$ as the primary dynamical variable for spatial evolution. Here we use the "dark Rabi frequency" $\Omega_{\mathrm{D}}$ via the Fleischhauer-Manka relation [15]: $\Omega_{\mathrm{D}}=-\mathrm{i}\left(\dot{\Omega}_{g} \Omega_{g^{\prime}}-\dot{\Omega}_{g^{\prime}} \Omega_{g}\right) / \Omega_{\mathrm{B}}^{2}$, which supplies the formula: $\theta(\zeta, \tau)=\mathrm{i} \int_{-\infty}^{\tau} \mathrm{d} \tau^{\prime} \Omega_{\mathrm{D}}\left(\zeta, \tau^{\prime}\right)$. Note that the formulae fix the origin and thereby eliminate the $\pm n \pi$ ambiguity in $\theta$ defined as the rotation angle in Eq. (3). Any $T$ following the passage of the pulses is effectively an infinite time, so we define the dark area as $\theta_{\mathrm{D}}(\zeta) \equiv \lim \left(T \gg \tau_{\mathrm{p}}\right) \theta(\zeta, T)$. We find that it obeys the nonlinear propagation law

$$
\frac{\partial \theta_{\mathrm{D}}}{\partial \zeta}=-\frac{\alpha}{2} \sin \left(\theta_{\mathrm{D}}+\phi\right)
$$

where $\phi$ is a constant determined by the initial (i.e. before the pulses come) ground-state coherence of the medium, and $\alpha$ is the normal inverse Beer length for an inhomogeneously broadened medium.

The analogy of Eq. (4) to the McCall-Hahn area theorem is obvious. Depending on initial conditions, there are infinitely many branch solutions for the dark area $\theta_{\mathrm{D}}$, as indicated in Fig. 1c. An area theorem does not specify pulse shapes, and we expect shapes to change as the pulses evolve to reach a stable area with matched forms, as is shown in Fig. 2. Note that the figure shows that, perhaps

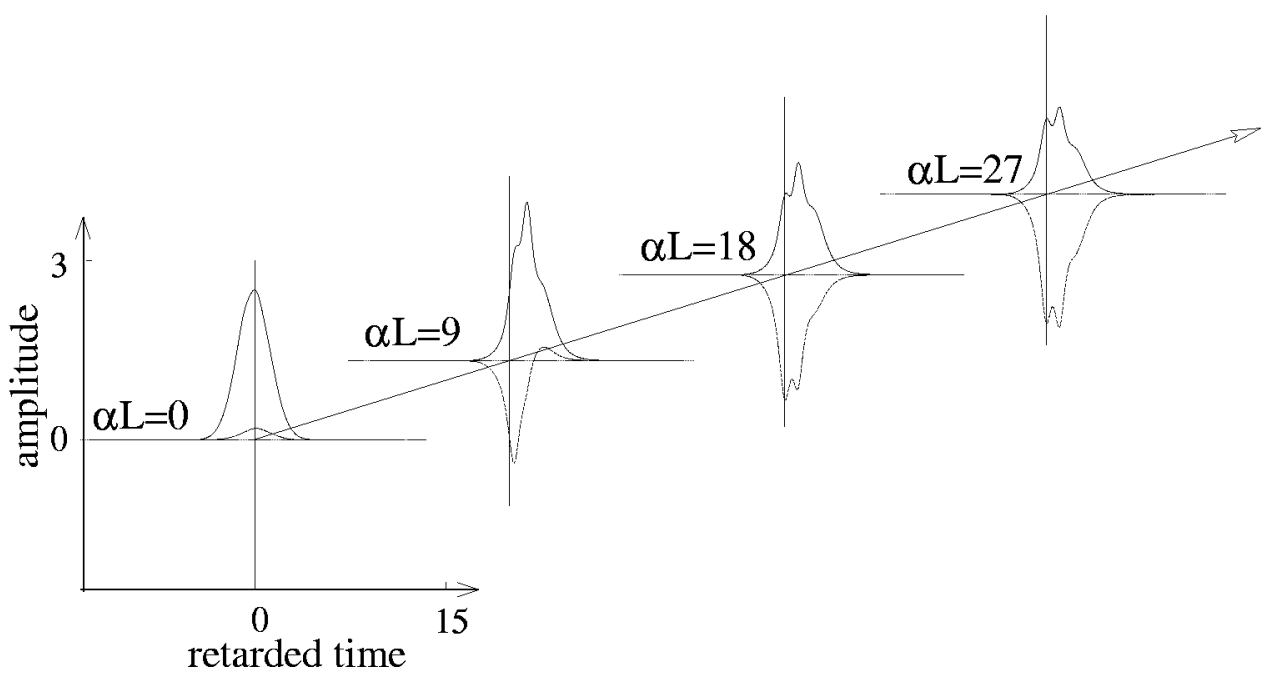

Fig. 2. Evolution plots showing the Rabi frequencies of the physical fields: $\Omega_{g}=$ $\left(2 d_{g} / \hbar\right) \mathcal{E}_{g}$ for the envelope $\mathcal{E}_{g}(\zeta, \tau)$ of the field that excites the ga transition, for example. Initially matched pulses with areas $6.2 \pi$ and $0.5 \pi$ propagate in a lambda medium with $\phi=2 \pi / 3$. Time is in units of inhomogeneous lifetime $T^{*}$ and the Rabi amplitudes in units of $\left(T^{*}\right)^{-1}$. 
unexpectedly, an initially matched pair of pulses does not remain matched, but may change dramatically before evolving into a stable final matching, which will not be at all similar to the initial matching.

We will report elsewhere [13] the details of the methods we have used to obtain Eq. (4). Intermediate results include a new wave equation for $\theta(\zeta, \tau)$ and spatial evolution equations for $D$ and $B$. The role of inhomogeneous broadening is emphasized.

\section{Acknowledgment}

This paper reports work presented in the Journal of Physics B Lecture at the conference Quantum Optics V, Kościelisko, Poland, June 2001. The work was partially supported by NSF grants PHY94-15583 and PHY00-72359, and the programme QUBITS of the European Commission.

\section{References}

[1] O.A. Kocharovskaya, Ya.I. Khanin, Pis'ma Zh. Eksp. Teor. Fiz. 48, 581 (1988); S.E. Harris, Phys. Rev. Lett. 62, 1033 (1989); M.O. Scully, S.-Y. Zhu, A. Gavrielides, ibid. 62, 2813 (1989).

[2] S.E. Harris, Physics Today 50, 36 (1997), and references therein.

[3] S.L. McCall, E.L. Hahn, Phys. Rev. Lett. 18, 908 (1967).

[4] L. Allen, J.H. Eberly, Optical Resonance and Two-Level Atoms, Wiley, New York 1975.

[5] J.H. Eberly, Opt. Express 2, 173 (1998).

[6] A. Rahman, J.H. Eberly, Opt. Express 4, 433 (1999).

[7] V.V. Kozlov, J.H. Eberly, Opt. Commun. 179, 85 (2000).

[8] G. Alzetta, A. Gozzini, L. Moi, G. Orriols, Nuovo Cimento B 36, 5 (1976); E. Arimondo, G. Orriols, Lett. Nuovo Cimento 17, 333 (1976); R.M. Whitley, C.R. Stroud, Jr., Phys. Rev. A 14, 1498 (1976).

[9] E. Arimondo, in: Progress in Optics, Ed. E. Wolf, Vol. XXXV, Elsevier, Amsterdam 1996, Ch. V, p. 259.

[10] J. Oreg, F.T. Hioe, J.H. Eberly, Phys. Rev. A 29, 690 (1984); C.E. Carroll, F.T. Hioe, Phys. Rev. A 42, 1522 (1990).

[11] S.E. Harris, Phys. Rev. Lett. 72, 52 (1994). See also J.H. Eberly, M.L. Pons, H.R. Haq, Phys. Rev. Lett. 72, 56 (1994).

[12] R. Grobe, F.T. Hioe, J.H. Eberly, Phys. Rev. Lett. 73, 3183 (1995).

[13] J.H. Eberly, V.V. Kozlov, in preparation.

[14] J.R. Kuklinski, U. Gaubatz, F.T. Hioe, K. Bergmann, Phys. Rev. A 40, 6741 (1989).

[15] M. Fleischhauer, A.S. Manka, Phys. Rev. A 54, 794 (1996). 